\title{
Implementation of a gait center training to improve walking ability and vital parameters in inpatient neurological rehabilitation- a cohort study
}

Stephanie Reichl ${ }^{1}$, Franz Weilbach ${ }^{1}$ and Jan Mehrholz $2,3^{*}$

\begin{abstract}
Background: Many studies showed that robot-assisted gait training might improve walking of patients after stroke. The question remains whether patients with other neurological diagnoses can improve their ability to walk by training in a gait center. Aim of the present study was therefore to investigate the effects of a gait center training in inpatient neurological rehabilitation on walking ability.

Methods: We implemented a gait center training in addition to individual inpatient rehabilitation. Our primary outcome was walking ability based on the Functional Ambulation Categories (FAC). Our secondary outcomes were vital capacity and blood pressure. We predefined subgroups of patients with ischemic and hemorrhagic stroke and critical illness myopathy (CIM) and polyneuropathy (CIP).

Results: We included 780 patients from our inpatient rehabilitation center in our cohort study. We analyzed 329 patients with ischemic, 131 patients with hemorrhagic stroke and 74 patients with CIP/ CIM.

A large number of patients were able to improve their ability to walk. At the end of rehabilitation, patients with ischemic stroke and FAC $3=$ increased theirFAC scores by $5 \%, F A C 4=4 \%$ and FAC $5=7 \%$. Patients with hemorrhagic stroke and FAC $3=$ increased by $5 \%, F A C 4=11 \%$ and FAC $5=9 \%$ and patients with CIP/CIM increased by FAC $3=3 \%$, FAC $4=22 \%$ and FAC $5=26 \%$.

The largest improvement in walking ability during rehabilitation had patients with a $F A C=1$ at baseline who improved by a median of 1.4 FAC points $(p<0.001)$. After adjusting for the number of gait training sessions, the largest improvement in walking ability during rehabilitation had patients with a FAC $=0$ at baseline who improved by 1.8 FAC points $(p<0.001)$.
\end{abstract}

Conclusions: Implementation of an additional gait center training may significantly improve walking ability in neurological rehabilitation.

Keywords: Stroke, CIP/CIM, Rehabilitation, Gait center training, Walking, Vital parameter

\footnotetext{
*Correspondence: Jan.mehrholz@tu-dresden.de; jan.mehrholz@klinik-

bavaria.de

${ }^{2}$ Department of Public Health, Dresden Medical School, Technical University Dresden, 01062 Dresden, Germany

${ }^{3}$ Wissenschaftliches Institut, Private Europäische Medizinische Akademie der

Klinik Bavaria in Kreischa GmbH, An der Wolfsschlucht 1-2, 01731 Kreischa, Germany

Full list of author information is available at the end of the article
}

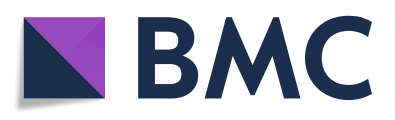

(- The Author(s). 2020 Open Access This article is licensed under a Creative Commons Attribution 4.0 International License, which permits use, sharing, adaptation, distribution and reproduction in any medium or format, as long as you give appropriate credit to the original author(s) and the source, provide a link to the Creative Commons licence, and indicate if changes were made. The images or other third party material in this article are included in the article's Creative Commons licence, unless indicated otherwise in a credit line to the material. If material is not included in the article's Creative Commons licence and your intended use is not permitted by statutory regulation or exceeds the permitted use, you will need to obtain permission directly from the copyright holder. To view a copy of this licence, visit http://creativecommons.org/licenses/by/4.0/. The Creative Commons Public Domain Dedication waiver (http://creativecommons.org/publicdomain/zero/1.0/) applies to the data made available in this article, unless otherwise stated in a credit line to the data. 


\section{Background}

Improvement of walking ability is a major goal in neurological rehabilitation [1]. It has been described that moderately intense physical activity in healthy adults is about 100 steps per minute. Patients with stroke, however, manage only one third of this, due to disease-related immobilization [2], and might therefore have reduced cardiopulmonary endurance [3, 4]. Therefore, many patients are usually only able to engage in moderately intensive physical activity for about 150 min per week [5]. Furthermore, patients with a neurological disorder such as a stroke have an increased risk of developing cardiovascular disease, a reinfarction, a heart attack or other heart problems due to such deconditioning and immobilization [2].

Gait disorders are one of the most common complaints in up to $60 \%$ of patients with cerebrovascular diseases [6]; and up to $80 \%$ of patients after stroke being affected [7]. It has been shown that aerobic capacity correlates with gait recovery $[8,9]$ and it is well known, that patients after stroke are limited in their activities of daily living such as walking due to reduced aerobic capacity [4]. It has been described that the cardiovascular fitness of patients after a stroke is no more than $50 \%$ of the performance level of healthy subjects [3]. Therefore improvement of cardiopulmonary fitness seems to be a prerequisite for good functional outcome and for the prevention of cardiorespiratory complications [10]. In order to reach such recommended level of physical activity, training in a gait center is used in neurological rehabilitation in addition to conventional gait rehabilitation. A gait center training consists of the elements standing and balancing trainer, robot-assisted gait training system and a treadmill with safety belt. With these devices, it is possible for patients to be mobilised vertically at an early stage, depending on their functional capacity and cardiopulmonary endurance [11]. Patients, who used the robot-assisted gait training, were able to walk of up to 1000 steps [12]. For instance many studies demonstrated the successful use of robotassisted gait training to increase physical activity of inpatient stroke patients [1, 13, 14]. Patients after stroke who are, however, unable to walk at the beginning of rehabilitation, defined as a Functional Ambulation Categories Score (FAC) of 0 to 2, benefited most from robotassisted gait training in terms of walking ability [1].

Until now it is not clear whether patients with other neurological diagnoses would improve their walking ability by training in a gait center in a similar way as patients after a stroke. Besides the development of walking ability, other questions seem to be important. For example, it is not yet clear whether the initial walking disability or the diagnosis influences the improvement of vital parameters by robot-assisted gait training in a gait center. Currently, only preliminary data are available on the use of treadmill training for patients who are unable to walk [15]. What we know is that neurological diseases may have an impact on static pulmonary volume and expiratory capacity [16]. Reduced lung ventilation leads to a reduced level of physical activity and increases the risk of broncho-pulmonary complications in neurological patients [17] and immobilised patients $[18,19]$. The maximum energy consumption of neurological patients averages 3.77 metabolic equivalents (MET) [20], while healthy people have between 8 to 10 MET [4]. An average MET of 3.77 seems, however, not sufficient to walk with a normal gait speed [20,21]. There is evidence that robot-assisted gait training might also improve cardiopulmonary endurance [8].

Daily training in a gait center in inpatient rehabilitation could therefore be useful in addition to early intensive mobilization for patients in neurological rehabilitation. In our view, however, it is time to investigate the effects of a gait training center training on walking ability, cardiopulmonary outcomes with a larger sample of patients.

The aim of the present study was, therefore, to investigate the effects of additional training in a gait center training on walking ability and vital capacity and blood pressure during inpatient neurological rehabilitation. Our main hypothesis was that walking ability and vital parameters improve.

\section{Methods \\ Protocol and registration}

We published a study protocol, which has been registered in the DRKS database under the ID DRKS00014090.

\section{Design}

This is a cohort study of patients being in their inpatient neurological rehabilitation.

\section{Participants}

We recruited all patients of the 'Klinik Bavaria' in Bad Kissingen, our neurological rehabilitation department, between July 2015 and June 2016 according to the following inclusion and exclusion criteria. Inclusion criteria were the ability to participate in the training program of our gait center.

Exclusion criteria were: 1) no gait training in gait center due to deterioration of general condition, a referral to a primary care clinic, isolation due to multi resistant germs and not able to be mobilised into a wheelchair and 2) contraindications to treatment with robot-assisted gait training system such as implantation of a cardiac pacemaker, artificial joint replacement in hip and knee joints up to two years after implantation, osteoporosis, manifested leg length difference or scoliosis or psychotic illnesses. The maximum weight limit of subjects was $150 \mathrm{~kg}$. 


\section{Setting}

All patients underwent their individual inpatient rehabilitation, including daily physiotherapy (e.g. lower leg strength, balance and gait training over ground), occupational therapy (e.g. hand-arm training) and speech therapy. As an add-on therapy patients received gait training center training for up to five days per week during their entire rehabilitation stay. The additional sessions in the gait center lasted up to $30 \mathrm{~min}$. As a main requirement for treatment in our gait center patients had to be able to sit in alone in a wheelchair.

\section{Intervention}

The therapeutic framework of our gait center, which consists of the elements standing and balancing trainers, robot-assisted gait training system and a treadmill with safety belt [11] has been steadily gaining acceptance in neurological rehabilitation. Therapy with these different devices enables patients to have social interaction and it also might increase their motivation to exercise since all these types of devices are available for each patient and they are able to train at the same time with other patients $[11,12]$.

According to the functional abilities at the beginning of rehabilitation, measured by means of Functional Ambulation Categories (FAC) [22-24], the patients trained with the most appropriate element in our gait center. E.g. patients with a FAC of 0 started their training in the gait center with the standing and balancing trainer. If patients with a FAC 0 to 2 were able to cope for $15 \mathrm{~min}$ in the standing and balancing trainer, they were trained with a robotic gait trainer [11].

The robot-assisted gait training in our gait center uses the end effector technology with two G-EO System Evolution and one G-EO System Basic (Reha Technology, Olten, Switzerland). The amount of activity on the robot-assisted gait training system varied between 50 to 1000 steps per session [25]. The therapeutic options for treatment in the robot-assisted gait training system included walking training in passive (G-EO System Basic), active assistive and active modes, and climbing stairs (G-EO System Evolution). The robot-assisted gait training system enables the regaining of motor coordination in a safe, intensive, task-specific and repetitive setting [10]. Therefore, this training setting is especially suitable for patients who are unable to walk. Participants in the gait center training sessions also performed standing balance training and treadmill training during the inpatient rehabilitation period and not just robotic gait training. E.g. if patients were unable to train with the robot-assisted gait system due to cardiopulmonary instability or reduced vigilance and trunk control, an alternative intervention was conducted using standing and balancing trainers (Thera Trainer, Hochdorf, Germany).

In this study, however, we analysed the number of gait training sessions through robotic-assisted gait training only.

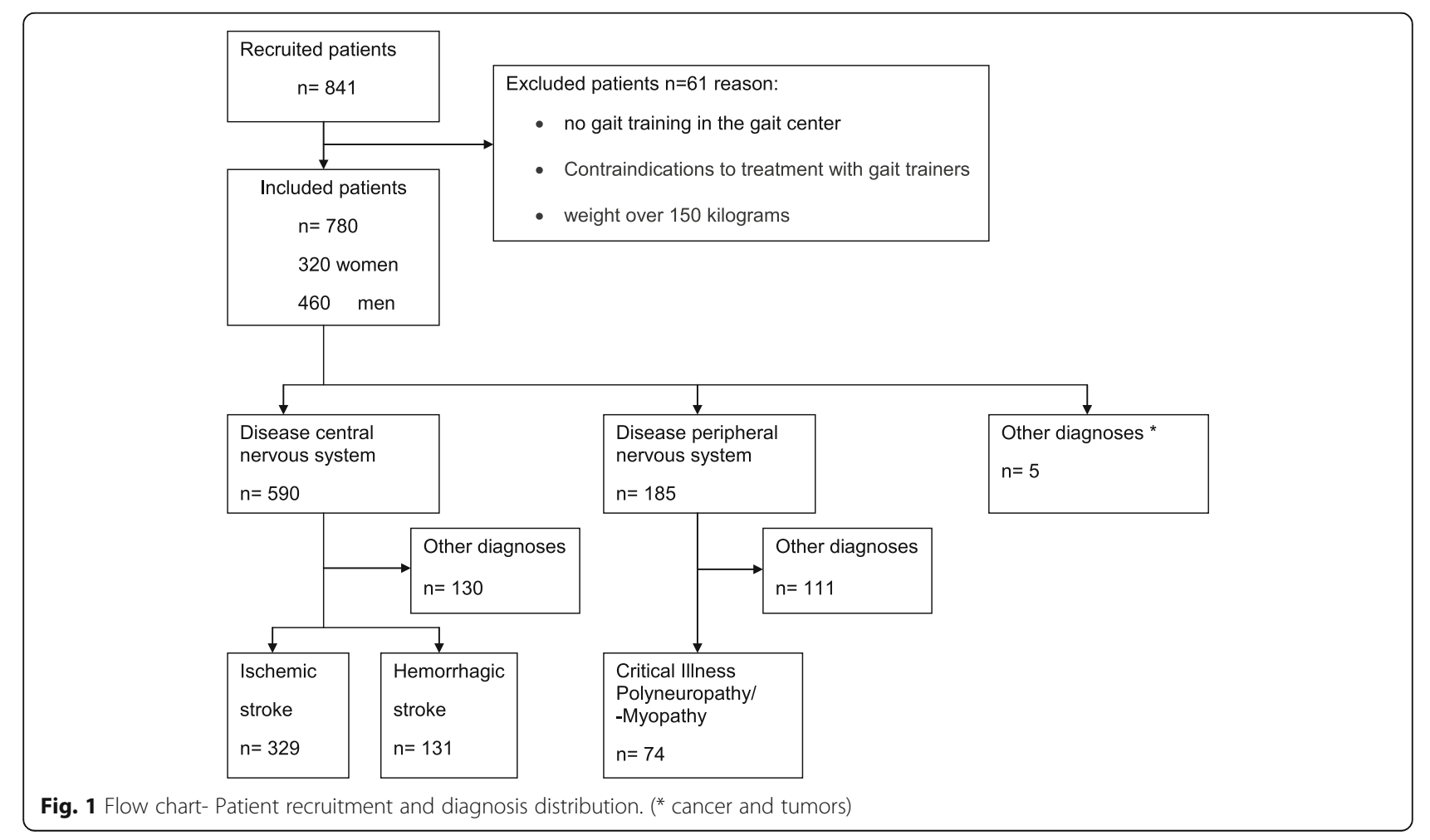




\section{Clinical assessments}

We used the Functional Ambulation Categories (FAC) as the primary outcome to measure walking ability from 0 (not able to walk) to 5 (independent walking ability) [22-24].

We used vital capacity (VC) as the secondary outcome, which was defined as the air volume of an exhalation (expiratory vital capacity EVC) [26]. We then recorded the volume between maximum inhalation and exhalation. We measured that volume by a pocket spirometer (spirotest, Riester, Jungingen) of the dry spirometer type [14], and recorded the EVC in $\mathrm{ccm}$. The pocket spirometer records an air volume of 1000 to $7000 \mathrm{ccm}$, divided into distances of $100 \mathrm{ccm}$. The readings of turbine spirometers were assumed to be about $5 \%$ below the exact value [16].

We used blood pressure (BP), as a secondary outcome which was measured daily by inpatient nursing staff with blood pressure cuffs (clinicus II- boso, Jungingen, Germany) and stethoscope manually indirectly to RivaRocci.

In patients with continuous monitoring, blood pressure was measured automatically (Infinity Gamma XL- Dräger, Lübeck, Germany; BSM-2301 K- NIHON KODEN, Rosbach v.d.H., Germany) and in patients without nursing care independently (medicus uno- boso, Jungingen, Germany) in mmHg. The daily recorded systolic and diastolic blood pressure readings were averaged over weekly averages for each week of rehabilitation stay per patient. We compared the values of all outcome measurements at the beginning of rehabilitation and at the end of rehabilitation in the context of this study.

\section{Statistical analysis}

We adhered to the SAMPL guidelines for statistical analysis [27]. As part of the descriptive data analysis, we calculated the measure of dispersion for the entire population as well as for the three selected subgroups. We considered the outcome data at the beginning of the rehabilitation as well as at the end of the rehabilitation. We assumed that there was a clear spread of the evaluated subgroup since no patient exclusion was based on age, disease duration or other characteristic features due to the study design. Therefore, we used non-parametric tests because we could not assume a normal distribution. We used medians with corresponding interquartile range (IQR) for plotting the development of the groups. We evaluated the steady dependent variables through paired Wilcoxon tests. In order to assess the improvement in walking ability, we calculated in a first analysis the gain in the FAC score as follows:

$F A C$ change $=(F A C$ score at end of inpatient rehabilitation) minus (FAC score at the start of inpatient rehabilitation).

In a second analysis we calculated an Analysis of Covariance (ANCOVA) and adjusted for the individual number of gait therapy sessions experienced by participantsusing the number of gait training sessions as covariate and computed least squares mean estimates for the FAC change, with 95\% Confidence interval for the

Table 1 Patient characteristics at baseline from total sample and selected subgroups

\begin{tabular}{|c|c|c|c|c|c|c|c|c|c|c|c|c|c|c|c|c|}
\hline & \multicolumn{2}{|c|}{$\begin{array}{l}\text { Total sample } \\
(n=780)\end{array}$} & \multicolumn{7}{|c|}{ Central Diagnoses } & \multirow{2}{*}{\multicolumn{3}{|c|}{$\begin{array}{l}\text { Peripheral } \\
\text { Diagnoses } \\
\text { CIPM }(n=74)\end{array}$}} & \multirow{2}{*}{\multicolumn{3}{|c|}{$\begin{array}{l}\text { NWA } \\
(n=89)\end{array}$}} & \multirow[t]{3}{*}{$\begin{array}{l}p \\
\text { value }\end{array}$} \\
\hline & \multirow[b]{2}{*}{ Median } & \multirow[b]{2}{*}{ IQR } & \multicolumn{3}{|c|}{ STI $(n=329)$} & \multicolumn{4}{|c|}{$\mathrm{STH}(n=131)$} & & & & & & & \\
\hline & & & $\mathrm{n}$ & Median & $\mathrm{IQR}$ & $n$ & Median & $\mathrm{IQR}$ & $\mathrm{n}$ & Median & IQR & $\mathrm{n}$ & Median & IQR & $\mathrm{n}$ & \\
\hline Age (years) & 68.0 & 22.0 & 780 & 72.0 & 18.0 & 329 & 72.0 & 23.0 & 131 & 71.0 & 14.8 & 74 & 72.0 & 8.0 & 89 & 0.496 \\
\hline duration of illness (weeks) & 4.3 & 16.6 & 629 & 3.0 & 5.6 & 284 & 3.9 & 10.8 & 109 & 9.0 & 10.5 & 51 & 3.7 & 6.7 & 73 & $\begin{array}{l}< \\
0.001\end{array}$ \\
\hline duration of rehabilitation (weeks) & 4.9 & 3.9 & 780 & 4.6 & 3.0 & 329 & 5.4 & 4.2 & 131 & 6.3 & 4.3 & 74 & 6.0 & 4.6 & 89 & $\begin{array}{l}< \\
0.001\end{array}$ \\
\hline $\begin{array}{l}\text { time rehabilitation beginning-start gait } \\
\text { trainer (weeks) }\end{array}$ & 0.7 & 1.0 & 616 & 0.7 & 0.9 & 266 & 0.7 & 1.5 & 102 & 1.7 & 3.5 & 43 & 3.4 & 5.7 & 26 & $\begin{array}{l}< \\
0.001\end{array}$ \\
\hline total gait trainer- sessions & 5.0 & 10.0 & 779 & 5.0 & 9.0 & 329 & 7.0 & 13.5 & 131 & 1.0 & 8.5 & 74 & 1.0 & 1.3 & 88 & $\begin{array}{l}< \\
0.001\end{array}$ \\
\hline FAC & 3 & 5 & 750 & 3 & 5 & 314 & 1 & 4 & 126 & 0 & 2 & 73 & 0 & 0 & 89 & $\begin{array}{l}< \\
0.001\end{array}$ \\
\hline Vital capacity (ccm) & 2100 & 1500 & 594 & 2100 & 1400 & 253 & 1900 & 1525 & 92 & 1600 & 1450 & 44 & 1300 & 800 & 40 & 0.052 \\
\hline Blood pressure systolic (mmHg) & 129 & 17 & 762 & 130 & 43 & 323 & 129 & 17 & 128 & 124 & 16 & 72 & 129 & 17 & 87 & $\begin{array}{l}< \\
0.001\end{array}$ \\
\hline Blood pressure diastolic $(\mathrm{mmHg})$ & 76 & 11 & 762 & 76 & 11 & 323 & 77 & 5 & 128 & 74 & 9 & 71 & 76 & 10 & 87 & 0.006 \\
\hline
\end{tabular}

STI ischemic stroke, STH hemorrhagic stroke, CIPM Critical Illness Polyneuropathy or -Myopathy, NWA patients who did not reach walking ability neither at the beginning nor at the end of rehabilitation- this subgroup $n=89$ is composed as follows $45(\mathrm{STI})+24(\mathrm{STH})+10(\mathrm{CIPM})+10$ (other neurological diagnosis), IQR interquartile range, FAC Functional Ambulation Categories, $p$ value- derived from paired Wilcoxon test 
adjusted mean FAC change $(95 \% \mathrm{CIs}$ for the least squared mean estimates),

The significance level was defined as alpha $=5 \%$ and we adjusted for alpha inflation with Bonferroni correction. All statistical analysis was done with the software $\mathrm{R}$ 3.3.2 and SAS 9.4 (SAS Institute Inc., Cary, NC, USA).

\section{Results}

\section{Patient characteristics}

We included 780 patients, among them 590 patients with central nervous system diseases, 185 patients with a disease of peripheral system and 5 patients with other diagnoses (cancer and tumors) (see Fig. 1, Flow chart).

We analyzed 329 patients with ischemic stroke (STI), 131 patients with hemorrhagic stroke (STH) and 74 patients with critical illness polyneuropathy or critical illness myopathy (CIPM). We found differences in patient characteristics between patients with central and peripheral neurological diagnoses. Patients with STH and STI received gait center therapy after a shorter period of illness (3 to 4 weeks) compared to other neurological diagnoses and the duration of rehabilitation was shorter (5 weeks). Patients with a peripheral diagnosis (CIPM) received gait center therapy at a later disease duration ( 9 weeks) and took longer to improve (6 weeks, as shown in Table 1$)$.

\section{Walking ability}

The percentage of participants with a walking ability of FAC $\geq 3$ increased from 53 to $75 \%$ during the rehabilitation process (see Fig. 2a). In the total sample, the largest improvement in walking ability during the rehabilitation process had patients with a $\mathrm{FAC}=1$ at baseline who improved by 1.4 FAC points $(p<0.001)$ (Table 2). After adjusting for the number of gait training sessions, in the total sample, the largest improvement in walking ability during rehabilitation had patients with a FAC $=0$ at baseline who improved by 1.8 FAC points $(p<0.001)$ (Table 3$)$.

Improvements of the ability to walk differed between patients with central and peripheral diagnosis (Tables 2 and 3).

In the subgroup STI, the percentage of participants with a walking ability of FAC $\geq 3$ increased from 55 to $71 \%$ during the rehabilitation process (see Fig. 2b). The largest improvement in walking ability during rehabilitation had patients with a baseline FAC of 1 who improved by 1.3 FAC points $(p=0.004)$ (as shown in Table 2). After adjusting for the number of gait training sessions, the largest improvement in walking ability during rehabilitation, had patients after STI and with a FAC $=0$ at baseline who improved by $2.1 \mathrm{FAC}$ points $(p<0.001)$ (as shown in Table 3).

In the subgroup $\mathrm{STH}$, the percentage of participants with a walking ability of FAC $\geq 3$ increased from 37 to $62 \%$ during rehabilitation (see Fig. 2c). Patients with a

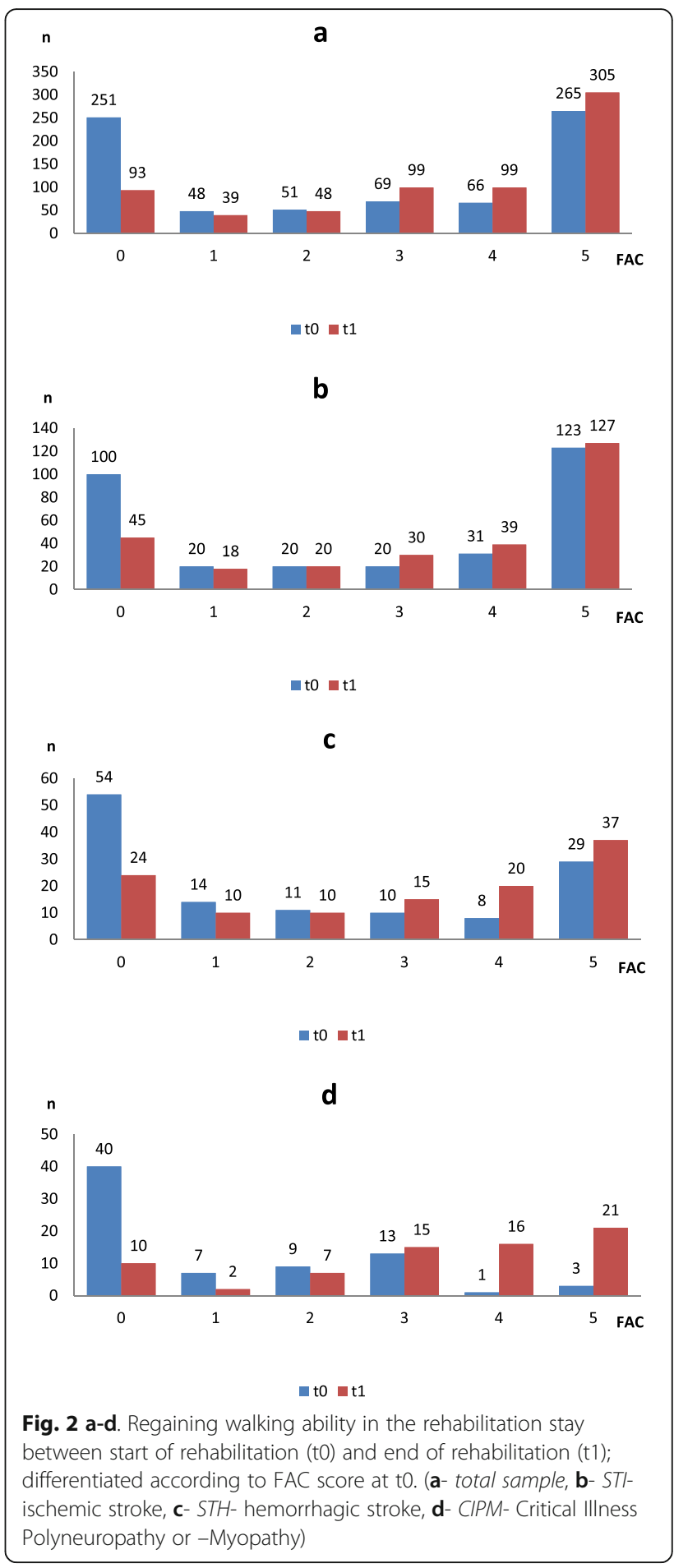

baseline FAC score of equal to 1, 2 and 3 had the largest gain of FAC score $(\mathrm{p}=0.004,0.002$ and 0.001 , respectively; as shown in Table 2).

After adjusting for the number of gait training sessions, the largest improvement in walking ability during rehabilitation, had patients after $\mathrm{STH}$ with a $\mathrm{FAC}=0$ at 
Table 2 Regaining walking ability, changes in Functional Ambulation Categories

\begin{tabular}{|c|c|c|c|c|c|c|c|c|c|c|c|c|}
\hline & \multirow{2}{*}{\multicolumn{3}{|c|}{ Total sample $(n=780)$}} & \multicolumn{6}{|c|}{ Central diagnoses } & \multirow{2}{*}{\multicolumn{3}{|c|}{$\begin{array}{l}\text { Peripheral diagnoses } \\
\text { CIPM }(n=74)\end{array}$}} \\
\hline & & & & \multicolumn{3}{|c|}{ STI $(n=329)$} & \multicolumn{3}{|c|}{$\mathrm{STH}(n=131)$} & & & \\
\hline & FC & IQR & $p$ value & $\overline{F C}$ & IQR & $p$ value & $\overline{F C}$ & IQR & $p$ value & $\overline{F C}$ & IQR & $p$ value \\
\hline \multicolumn{13}{|c|}{ FAC at t0 } \\
\hline 0 & $1.0^{*}$ & 2.0 & $<0.001$ & 0.3 & 1.6 & $<0.001$ & 0.4 & 1.9 & $<0.001$ & $1.8^{*}$ & 3.1 & $<0.001$ \\
\hline 1 & $1.4^{*}$ & 2.4 & $<0.001$ & $1.3^{*}$ & 2.2 & 0.004 & $1.5^{*}$ & 3.4 & & $2.2^{*}$ & 2.2 & 0.021 \\
\hline 2 & $1.0^{*}$ & 1.0 & $<0.001$ & $1.0^{*}$ & 1.0 & 0.002 & $1.5^{*}$ & 1.1 & 0.006 & $2.4^{*}$ & 1.8 & 0.013 \\
\hline 3 & $1.0^{*}$ & 1.7 & $<0.001$ & $1.0^{*}$ & 1.3 & 0.001 & $1.5^{*}$ & 0.8 & 0.013 & $1.3^{*}$ & 0.9 & 0.002 \\
\hline 4 & 0.4 & 1.0 & $<0.001$ & 0.0 & 1.0 & $<0.001$ & $1.1^{*}$ & 0.8 & 0.019 & 0.9 & 0.0 & 1.000 \\
\hline 5 & & & & & & & & & 0.037 & & & \\
\hline
\end{tabular}

FAC Functional Ambulation Categories,

FC FAC change- Median Gain in walking ability between t0(start of rehabilitation) and $\mathrm{t} 1$ (end of inpatient rehabilitation), $I Q R$ interquartile range from $\mathrm{FC}$,

$p$ value- derived from paired Wilcoxon test- ${ }^{*} p<0.05$ and clinically relevant (minimal improvement by one FAC point),

$S T I$ ischemic stroke,

STH hemorrhagic stroke,

CIPM Critical Illness Polyneuropathy or -Myopathy

baseline who improved by 1.9 FAC points $(p<0.001)$ (as shown in Table 3).

Furthermore, in the subgroup CIPM, the percentage of participants with a walking ability of FAC $\geq 3$ increased from 23 to $74 \%$ during the rehabilitation process (see Fig. 2d). Patients with CIPM and baseline FAC scores of 3 had the largest gain of FAC score by 2.4 FAC points $(p=0.013$, as shown in Table 2).

After adjusting for the number of gait training sessions, the largest improvement in walking ability during rehabilitation, had patients after CIPM with a $\mathrm{FAC}=0$ at baseline who improved by 1.7 FAC points $(p<0.001)$ (as shown in Table 3).

In total 89 patients did not reach walking ability, $79 \mathrm{pa}-$ tients from the subgroups $(\mathrm{STI}=45, \mathrm{STH}=24, \mathrm{CIPM}=$
10) and ten patients with other neurological diagnoses (Table 5).

As shown in Fig. 2a-d a number of patients dropped out until the end of rehabilitation, due to medical reasons, transfer back to acute hospital, palliative treatment or early discharge to a nursing home.

\section{Vital capacity}

The mean vital capacity (VC) improved by $300 \mathrm{ccm}(p<$ 0.001 ) in the total sample with a baseline FAC of 0 . The mean VC of Patients with a baseline FAC of 1 to 5, however, did not improved significantly.

In patients in the STI and STH subgroup, who had a FAC of 0 at baseline, VC improved significantly by $200 \mathrm{ccm}$ $(p=0.02)$ and $500 \mathrm{ccm}(p=0.001)$, respectively (Table 4$)$.

Table 3 Regaining walking ability, changes in Functional Ambulation Categories adjusted by the number of gait training sessions

\begin{tabular}{|c|c|c|c|c|c|c|c|c|c|c|c|c|}
\hline & \multirow{2}{*}{\multicolumn{3}{|c|}{ Total sample $(n=780)$}} & \multicolumn{6}{|c|}{ Central diagnoses } & \multirow{2}{*}{\multicolumn{3}{|c|}{$\frac{\text { Peripheral diagnoses }}{\text { CIPM }(n=74)}$}} \\
\hline & & & & \multicolumn{3}{|c|}{ STI $(n=329)$} & \multicolumn{3}{|c|}{$\mathrm{STH}(n=131)$} & & & \\
\hline & FCadj & $95 \% \mathrm{Cl}$ & $\overline{p \text { value }}$ & FCadj & $95 \% \mathrm{Cl}$ & $p$ value & FCadj & $95 \% \mathrm{Cl}$ & $\overline{p \text { value }}$ & FCadj & $95 \% \mathrm{Cl}$ & $p$ value \\
\hline \multicolumn{13}{|c|}{ FAC at t0 } \\
\hline 0 & $1.8^{*}$ & $1.7 ; 2.0$ & $<0.001$ & $2.1^{*}$ & $1.9 ; 2.3$ & $<0.001$ & $1.9^{*}$ & $1.8 ; 2.1$ & $<0.001$ & $1.7^{*}$ & $1.5 ; 1.8$ & $<0.001$ \\
\hline 1 & $1.5^{*}$ & $1.2 ; 1.9$ & $<0.001$ & $1.6^{*}$ & $1.2 ; 2.1$ & $<0.001$ & $1.6^{*}$ & $1.2 ; 2.0$ & $<0.001$ & $1.4^{*}$ & $1.0 ; 1.7$ & $<0.001$ \\
\hline 2 & $1.2^{*}$ & $0.9 ; 1.5$ & $<0.001$ & $1.4^{*}$ & $0.9 ; 1.8$ & $<0.001$ & $1.2^{*}$ & $0.8 ; 1.5$ & $<0.001$ & $1.0^{*}$ & $0.7 ; 1.3$ & $<0.001$ \\
\hline 3 & 0.9 & $0.6 ; 1.2$ & $<0.001$ & 0.8 & $0.5 ; 1.2$ & $<0.001$ & 0.9 & $0.6 ; 1.2$ & $<0.001$ & 0.8 & $0.5 ; 1.1$ & $<0.001$ \\
\hline 4 & 0.5 & $0.2 ; 0.8$ & $<0.001$ & 0.5 & $0.1 ; 0.9$ & 0.048 & 0.5 & $0.2 ; 0.8$ & 0.013 & 0.5 & $0.2 ; 0.8$ & 0.006 \\
\hline
\end{tabular}

FAC- Functional Ambulation Categories,

FCadj FAC change adjusted by the number of gait training sessions received - adjusted mean gain in walking ability between to(start of rehabilitation) and t 1 (end of inpatient rehabilitation) revealed by Analysis of Covariance (ANCOVA) using number of gait training sessions as co-variate and computation of least squares mean estimates for the classes FAC at t0,

$95 \% \mathrm{Cl}-95 \%$ Confidence interval for the adjusted mean FC $(95 \% \mathrm{Cls}$ for the least squares mean estimates for the classes $\mathrm{FAC}$ at t0),

$p$ value- derived from ANCOVA least squares estimates for FAC at t0, ${ }^{*} p<0.05$ and clinically relevant (minimal improvement by one $\mathrm{FAC}$ point),

STI ischemic stroke,

STH hemorrhagic stroke,

CIPM Critical Illness Polyneuropathy or -Myopathy 
Table 4 Development vital capacity

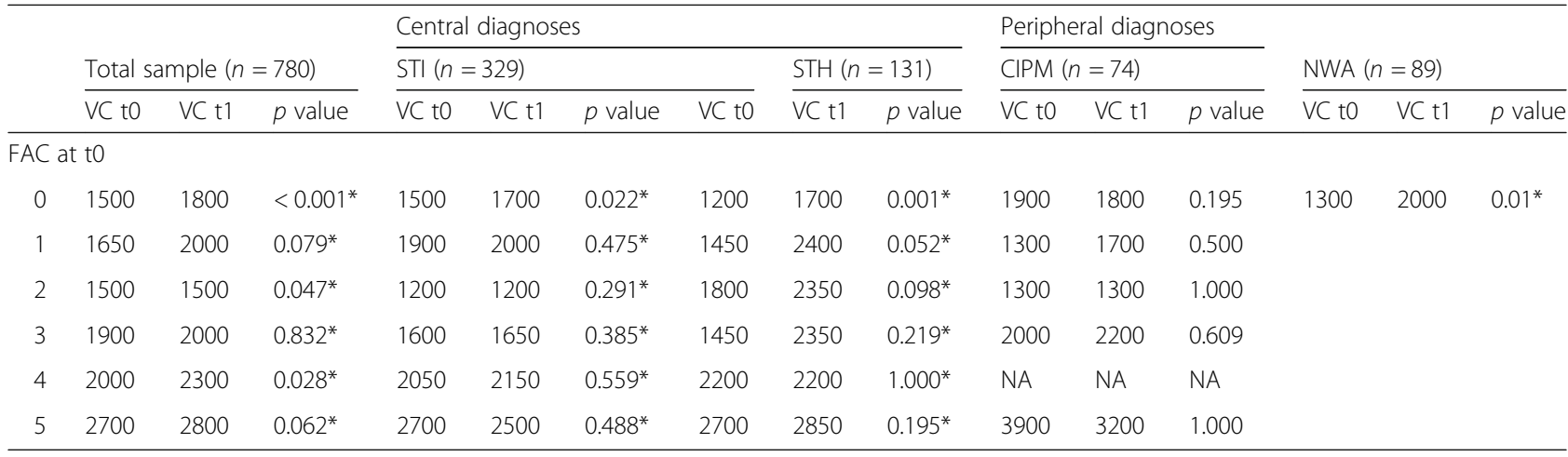

FAC Functional Ambulation Categories, VC Median vital capacity in Cubic centimeter (ccm), to- start of inpatient rehabilitation, $t 1$ - end of inpatient rehabilitation, $p$ value- derived from paired Wilcoxon test- ${ }^{*} p<0.05$, STI ischemic stroke, STH hemorrhagic stroke, CIPM Critical Illness Polyneuropathy or -Myopathy, NWA patients who did not reach walking ability neither at the beginning nor at the end of rehabilitation- this subgroup $n=89$ is composed as follows $45(\mathrm{STI})+24(\mathrm{STH})+$ $10($ CIPM) + 10(other neurological diagnosis)

In patients with STI and STH who regained walking function, and patients with CIPM, however, VC did not improve significantly.

\section{Blood pressure}

The blood pressure, BP, in the total sample was at baseline already within a normal range with a systolic BP of $129 \mathrm{mmHg}$ and diastolic BP of $76 \mathrm{mmHg}$. No significant improvement of BP during rehabilitation was found (systolic BP of $127 \mathrm{mmHg}$ and diastolic BP of $76 \mathrm{mmHg}$ at the end of rehabilitation, $p=0.05$ and $p=0.38$, respectively). There were no differences neither for systolic nor for diastolic BP between the subgroups STI, STH and CIPM during rehabilitation (Table 5).

\section{Discussion}

The present prospective cohort study is the first study to examine a relatively large sample of 780 patients during inpatient rehabilitation in a gait center. It is one of the first studies in neurological rehabilitation that evaluates the improvement of walking ability by comparing different diagnosis without excluding several comorbidities. The results of this study are therefore be applicable to inpatient neurological rehabilitation.

We claimed that walking ability and vital parameters can be improved with gait center training. In addition, we hypothesized that the training improves or stabilizes the vital parameters of patients who are unable to walk in the rehabilitation process.

As a main result, we found that gait training can improve walking ability and provides the greatest benefit to specific patient subgroups e.g. patients after stroke. Gait center training seems to be most effective for nonambulatory patients with an initial walking ability of FAC equal to 1. After adjusting for the number of walking training sessions, we found that patients with $\mathrm{FAC}$ equal to 0 in particular benefit most from gait center therapy.

A recent Cochrane Review suggested recently robotassisted gait training might be effectivefor non-ambulatory

Table 5 Development blood pressure

\begin{tabular}{|c|c|c|c|c|c|c|c|c|c|c|c|c|c|c|c|}
\hline & \multirow{2}{*}{\multicolumn{3}{|c|}{ Total sample $(n=780)$}} & \multicolumn{6}{|c|}{ Central diagnoses } & \multicolumn{6}{|c|}{ Peripheral diagnoses } \\
\hline & & & & \multicolumn{4}{|c|}{ STI $(n=329)$} & \multicolumn{2}{|c|}{$\mathrm{STH}(n=131)$} & \multicolumn{3}{|c|}{$\overline{\mathrm{CIPM}}(n=74)$} & \multicolumn{3}{|c|}{ NWA $(n=89)$} \\
\hline & BP t0 & $\mathrm{BP}$ t1 & $p$ value & BP t0 & BP t1 & $p$ value & BP t0 & $\mathrm{BP}$ t1 & $p$ value & BP t0 & BP t1 & $p$ value & BP t0 & BP t1 & $p$ value \\
\hline \multicolumn{16}{|c|}{$\overline{F A C}$ at t0 } \\
\hline 0 & $128 / 75$ & $124 / 75$ & $0.08 / 0.68$ & $130 / 74$ & $126 / 76$ & $0.44 / 0.32$ & $131 / 78$ & $124 / 74$ & $0.04 / 0.07$ & $123 / 74$ & $123 / 73$ & $0.91 / 0.76$ & $129 / 76$ & $124 / 74$ & $0,06 / 0,01$ \\
\hline 1 & $127 / 75$ & $126 / 76$ & $0.37 / 0.56$ & $126 / 74$ & $128 / 72$ & $0.42 / 0.84$ & $127 / 77$ & $124 / 77$ & $0.51 / 0.88$ & $139 / 77$ & $131 / 72$ & $0.38 / 0.07$ & & & \\
\hline 2 & $124 / 76$ & $127 / 75$ & $0.43 / 0.99$ & $133 / 77$ & $130 / 75$ & $0.46 / 0.17$ & $121 / 74$ & $128 / 78$ & $0.44 / 0.24$ & $117 / 73$ & $127 / 73$ & $0.04 / 0.40$ & & & \\
\hline 3 & $128 / 75$ & $126 / 75$ & $0.69 / 0.83$ & $129 / 75$ & $130 / 76$ & $0.34 / 0.22$ & $124 / 74$ & $121 / 74$ & $0.95 / 0.86$ & $131 / 72$ & $126 / 76$ & $0.91 / 0.61$ & & & \\
\hline 4 & $129 / 78$ & $129 / 77$ & $0.34 / 0.87$ & $128 / 76$ & $127 / 74$ & $0.07 / 0.29$ & $134 / 78$ & $129 / 80$ & $1.00 / 0.56$ & $105 / 64$ & $111 / 68$ & $1.00 / 1.00$ & & & \\
\hline 5 & $130 / 80$ & $130 / 80$ & $0.10 / 0.16$ & $133 / 80$ & $132 / 79$ & $0.01 / 0.01$ & 130/80 & 130/80 & $0.98 / 0.43$ & $129 / 80$ & $121 / 85$ & $0.50 / 0.50$ & & & \\
\hline
\end{tabular}

FAC Functional Ambulation Categories, BP Median blood pressure in millimeters of mercury $(\mathrm{mmHg})$, t0- start of inpatient rehabilitation, $t 1$ - end of inpatient rehabilitation, $p$ value- derived from paired Wilcoxon test- ${ }^{*} p<0.05$ and clinically relevant, STI ischemic stroke, STH hemorrhagic stroke, CIPM Critical IIIness Polyneuropathy or -Myopathy, NWA patients who did not reach walking ability neither at the beginning nor at the end of rehabilitation- this subgroup $n=89$ is composed as follows $45(\mathrm{STI})+24(\mathrm{STH})+10(\mathrm{CIPM})+10$ (other neurological diagnosis) 
patients (FAC 0 to 2) [1]. Our results are in this line with a recommendation for gait center training especially for patients with an initial FAC Score of 0-2.

A recent network-meta-analysis found that robotassisted gait training after stroke might be effective if end-effectors are used [12]. Our study supports the argument that even non-ambulatory patients with an FAC of 0 to 2 could benefit and improve their walking ability if end-effector gait training is applied.

We could not confirm our hypothesis that patients who were able to walk in the rehabilitation process would also improve in vital parameters. Our results shows, however, that the vital capacity of those patients who are unable to walk at rehabilitation onset might improve. This effect was consistent in all diagnostic groups. The results are somewhat in contrast to the literature who described that robot-assisted gait and treadmill training has the potential to produce aerobic exercise for patients with 'limited and incapable disease' and in patients who are already able to walk $[2,15]$.

As described in the literature we observed in patients after stroke, impaired breathing due to weakened respiratory muscles and reduced chest mobility when breathing in and out [28]. Especially nonambulatory patients spend much time of the day sitting in a wheelchair which might reinforce this problem. Additional training in the gait centre could interrupt such a vicious circle of physical inactivity, unfavorable immobilized position and reduced lung volume and our results shows effects on $\mathrm{VC}$ for patients with FACs equal to 0 .

Oxygen demand during walking is greater in patients with ischemic stroke than in healthy controls [29]. In order to meet this demand, a sufficiently large gas exchange is necessary. If this cannot be provided e.g. due to decreased chest mobility and reduced lung volumes, walking distances might be shorter than necessary.

Additional mobilization through gait center training could increase the cardiopulmonary load and thus promote breathing frequency and respiratory effort [30].

\section{Limitations}

This study has some potential limitations. First, this was a single-center study and it needs a reevaluation in a multicenter design. Second, this was just an observational study and no causal relationship can be drawn. This study is therefore more likely to be seen as a feasibility analysis of a relatively large cohort of patients in neurological inpatient rehabilitation.

We analysed to what extent the gait center training can be used appropriately with the available equipment capacities. In this study, we found that in some cases the patients remained well below the target of five training applications. This is due to drop outs such as acute treatment or deterioration of the patients' general condition.
Future studies should therefore aim to reduce the discrepancy between target and effective therapies by developing appropriate prognostic parameters.

The effects on walking ability as well as vital capacity result from a combination of physiotherapy together with all other components from the gait center such as therapies in the standing trainer and on the treadmill, robot-assisted gait training and all other treatment components in an individually adapted rehabilitation. The improvements showed can therefore not only be attributed to the robot-assisted gait therapy, but are also due to a consistent early mobilization into a vertical position. We focused in this study, however, only on the number of robotic-gait training sessions and used this as an important factor when adjusting our results.

Another limitation in this study is that only gait ability, $\mathrm{VC}$ and BP was measured. In future studies, activities of daily life, physical activity, walking ability at home and quality of life should also be measured.

It should be considered that the use of a turbine pocket spirometer to measure $\mathrm{VC}$ during expiration is less meaningful than the use of a pneumotachograph. Since the measurement is not a purely mechanical determination of the measured values, neither a respiratory curve nor detailed measurement results are recorded digitally. The determination of the coarse value can only be read off visually by the measuring personnel on the spirometer scale at $100 \mathrm{ml}$ intervals.

\section{Conclusions}

The present study showed for the first time in a large cohort study in inpatient neurological rehabilitation the clinical effects of an additional gait center training on walking ability.

\section{Abbreviations}

BP: Blood pressure; ccm: Cubic centimeter; CIPM: Critical Illness Polyneuropathy or -Myopathy; EVC: Expiratory vital capacity; FAC: Functional Ambulation Categories; IQR: Interquartile range; MET: Metabolic equivalents; mmHg: Millimeters of mercury; STH: Hemorrhagic stroke; STI: Ischemic stroke; VC: Vital capacity

\section{Acknowledgments \\ The authors would like to acknowledge the physiotherapy team Neurology of the Klinik Bavaria Bad Kissingen for their assistance in the data collection.}

\section{Authors' contributions}

Stephanie Reichl (SR) contributed towards conceptualization of the study, data collection, processing and analysis as well as drafting and subsequent revisions of the manuscript and contributed to and approved the final manuscript of the study. Franz Weilbach (FW) contributed towards conceptualization of the study, data analysis as well as drafting and subsequent revisions of the manuscript and contributed to and approved the final manuscript of the study. Jan Mehrholz (JM) contributed towards conceptualization of the study, data processing and analysis as well as drafting and subsequent revisions of the manuscript and contributed to and approved the final manuscript of the study. 


\section{Funding}

This work was supported by the 'Klinik Bavaria' Bad Kissingen. JM is supported by the 'Wissenschaftliches Institut- Private Europäische Medizinische Akademie der Klinik Bavaria in Kreischa GmbH'. We acknowledge support by the Open Access Publication Funds of the SLUB/TU Dresden.

\section{Availability of data and materials}

The datasets supporting the conclusions of this article are included within the article, figures and tables.

\section{Ethics approval and consent to participate}

The study was conducted with the approval of the local ethics committee of the University of Applied Sciences Gera Germany (project number: 112010301) and registered in the German Register of Clinical Trials with the ID: DRKS00014090 (a registry that is a main registry of the WHO International Clinical Trials Registration Platform (ICTRP)).

\section{Consent for publication}

Standardized data were evaluated anonymously.

\section{Competing interests}

The authors declare that they have no competing interests.

\section{Author details}

${ }^{1}$ Klinik Bavaria Bad Kissingen, Von-der-Tann-Straße 18- 22, 97688 Bad Kissingen, Germany. 'Department of Public Health, Dresden Medical School, Technical University Dresden, 01062 Dresden, Germany. ${ }^{3}$ Wissenschaftliches Institut, Private Europäische Medizinische Akademie der Klinik Bavaria in Kreischa GmbH, An der Wolfsschlucht 1-2, 01731 Kreischa, Germany.

Received: 26 March 2019 Accepted: 27 February 2020

Published online: 04 March 2020

\section{References}

1. Mehrholz J, et al. Electromechanical-assisted training for walking after stroke. Cochrane Database Syst Rev. 2017;5:CD006185

2. Han EY, et al. Robot-assisted gait training improves brachial-ankle pulse wave velocity and peak aerobic capacity in subacute stroke patients with totally dependent ambulation: randomized controlled trial. Medicine (Baltimore). 2016;95(41):e5078.

3. Macko RF, et al. Treadmill exercise rehabilitation improves ambulatory function and cardiovascular fitness in patients with chronic stroke: a randomized, controlled trial. Stroke. 2005;36(10):2206-11.

4. Billinger SA, et al. Reduced cardiorespiratory fitness after stroke: biological consequences and exercise-induced adaptations. Stroke Res Treat. 2012; 2012:959120.

5. English C, et al. Physical activity and sedentary behaviors in people with stroke living in the community: a systematic review. Phys Ther. 2014;94(2): 185-96.

6. Paolucci S, et al. Quantification of the probability of reaching mobility independence at discharge from a rehabilitation hospital in nonwalking early ischemic stroke patients: a multivariate study. Cerebrovasc Dis. 2008; 26(1):16-22.

7. Kang CG, et al. Views of physiatrists and physical therapists on the use of gait-training robots for stroke patients. J Phys Ther Sci. 2016;28(1):202-6.

8. Chang WH, et al. Effects of robot-assisted gait training on cardiopulmonary fitness in subacute stroke patients: a randomized controlled study. Neurorehabil Neural Repair. 2012;26(4):318-24.

9. Outermans JC, et al. Effects of a high-intensity task-oriented training on gait performance early after stroke: a pilot study. Clin Rehabil. 2010;24(11):979_ 87.

10. Lee SY, et al. Can lowering the guidance force of robot-assisted gait training induce a sufficient metabolic demand in subacute dependent ambulatory patients with stroke? Arch Phys Med Rehabil. 2017:98(4):695-700.

11. Hesse $\mathrm{S}$, et al. Loco-studio: an effective and efficiant locomotion group therapy approach in patients of Phase B,C and D in neuro-rehabilitation. Neurol Rehabil. 2015;21(4):195-200.

12. Mehrholz J, et al. The improvement of walking ability following stroke. Dtsch Arztebl Int. 2018;115(39):639-45.
13. Pohl $M$, et al. Repetitive locomotor training and physiotherapy improve walking and basic activities of daily living after stroke: a single-blind, randomized multicentre trial (DEutsche GAngtrainerStudie, DEGAS). Clin Rehabil. 2007;21(1):17-27.

14. Bruni MF, et al. What does best evidence tell us about robotic gait rehabilitation in stroke patients: a systematic review and meta-analysis. J Clin Neurosci. 2018:48:11-7.

15. Munari $D$, et al. High-intensity treadmill training improves gait ability, VO2peak and cost of walking in stroke survivors: preliminary results of a pilot randomized controlled trial. Eur J Phys Rehabil Med. 2018;54(3):408-18.

16. Quanjer PH, et al. Lung volumes and forced ventilatory flows. Eur Respir J. 1993;6(Suppl 16):5-40.

17. Brogan $E$, et al. Respiratory infections in acute stroke: nasogastric tubes and immobility are stronger predictors than dysphagia. Dysphagia. 2014;29(3): 340-5.

18. Loeb M, et al. Risk factors for pneumonia and other lower respiratory tract infections in elderly residents of long-term care facilities. Arch Intern Med. 1999;159(17):2058-64.

19. Wu X, et al. The association between major complications of immobility during hospitalization and quality of life among bedridden patients: a 3 month prospective multi-center study. PLoS One. 2018;13(10):e0205729.

20. Rimmer JH. Exercise and physical activity in persons aging with a physical disability. Phys Med Rehabil Clin N Am. 2005;16(1):41-56.

21. Lefeber N, Swinnen E, Kerckhofs $E$. The immediate effects of robotassistance on energy consumption and cardiorespiratory load during walking compared to walking without robot-assistance: a systematic review. Disabil Rehabil Assist Technol. 2017;12(7):657-71.

22. Holden MK, Gill KM, Magliozzi MR. Gait assessment for neurologically impaired patients. Standards for outcome assessment. Phys Ther. 1986; 66(10):1530-9.

23. Collen FM, Wade DT, Bradshaw CM. Mobility after stroke: reliability of measures of impairment and disability. Int Disabil Stud. 1990;12(1):6-9.

24. Wade DT. Measurement in neurological rehabilitation. Curr Opin Neurol Neurosurg. 1992;5(5):682-6

25. Esquenazi A, Packel A. Robotic-assisted gait training and restoration. Am J Phys Med Rehabil. 2012;91(11 Suppl 3):S217-27 quiz S228-31.

26. Criée CP, et al. Standardization of spirometry: 2015 Update. Published by German Atemwegsliga, German respiratory society and German Society of Occupational and Environmental Medicine. Pneumologie. 2015;69(3):147-64.

27. Lang TA, Altman DG. Basic statistical reporting for articles published in biomedical journals: the "statistical analyses and methods in the published literature" or the SAMPL quidelines. Int J Nurs Stud. 2015;52(1):5-9.

28. Ezeugwu VE, et al. Comparative lung function performance of stroke survivors and age-matched and sex-matched controls. Physiother Res Int 2013;18(4):212-9.

29. Delussu AS, et al. Physiological responses and energy cost of walking on the gait trainer with and without body weight support in subacute stroke patients. J Neuroeng Rehabil. 2014;11:54.

30. Awad $L N$, et al. Paretic propulsion and trailing limb angle are key determinants of long-distance walking function after stroke. Neurorehabil Neural Repair. 2015;29(6):499-508.

\section{Publisher's Note}

Springer Nature remains neutral with regard to jurisdictional claims in published maps and institutional affiliations.

Ready to submit your research? Choose BMC and benefit from

- fast, convenient online submission

- thorough peer review by experienced researchers in your field

- rapid publication on acceptance

- support for research data, including large and complex data types

- gold Open Access which fosters wider collaboration and increased citations

- maximum visibility for your research: over $100 \mathrm{M}$ website views per year

At BMC, research is always in progress.

Learn more biomedcentral.com/submissions 